\section{Evidenz und eigene Erfahrung zählen}

- Viele Faktoren beeinflussen das Verordnungsverhalten von Ärzten. Neben Wirksamkeit und Verträglichkeit spielen auch die Erfahrung des Arztes, die Erwartungshaltung der Patienten oder Kosten eine Rolle. Eine Forderung, die in jüngerer Zeit an Gewicht gewonnen hat, ist die Evidenzbasierung der getroffenen Maßnahme.

Welchen Stellenwert diese Forderung für Ärzte in der Praxis tatsächlich hat, was sie darunter verstehen und ob sie diese Maßstäbe auch an die Verordnung von Phytotherapeutika anlegen, war Gegenstand einer Erhebung des Unternehmens coliquio.

\section{Wirksamkeit steht an erster Stelle} Um herauszufinden, welches die primären Kriterien für eine Therapieentscheidung sind, wurden exemplarisch 120 Allgemeinmedizinern und HNO-Ärzten zunächst allgemeine Fragen zu Arzneimitteln und dem persönlichen Verordnungsverhalten gestellt. Aus den Antworten wurde deutlich: Das mit Abstand wichtigste Kriterium für die Verordnung oder Empfehlung eines Arzneimittels ist dessen Wirksamkeit (74\%), gefolgt von seiner Verträglichkeit (51\%).

\section{Erfahrung und Studien - beides zählt}

Doch wie lässt sich aus Sicht der Ärzte Wirksamkeit nachweisen? Welche Faktoren halten sie für entscheidend? Eine wichtige Rolle spielen hier klinische (placebokontrollierte, doppelblinde) Studien. Für knapp ein Viertel der Befragten sind sie das ausschlaggebende Merkmal. 15\% verlassen sich lieber auf ihre eigenen positiven $\mathrm{Er}$ fahrungen. Für die Mehrheit ist jedoch die Kombination aus beidem der entscheidende Maßstab. Entsprechend hielten 43\% der Teilnehmer eine Arznei dann für wirksam, wenn es Belege aus Wissenschaft und eigener Erfahrung gibt.

David Sackett, einer der Urväter der evidenzbasierten Medizin (EbM), würde sich über dieses Ergebnis freuen, entspricht die Einschätzung der Ärzte doch weitgehend seinem Konzept von EbM: Die Integration individueller klinischer Expertise (Arzterfahrung) mit der bestmöglichen externen Evidenz aus systematischer Forschung. tät und Datenlage stimmen.

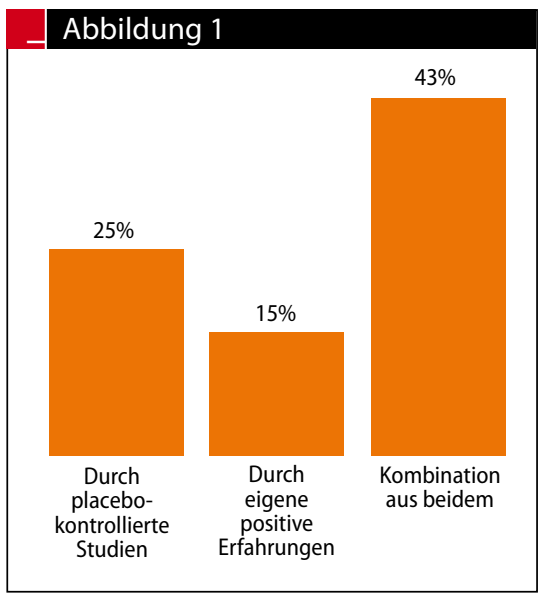

Abb. 1 Ärzte setzen auf Studien und eigene Erfahrung. Auf die Frage „Wie lässt sich die Wirksamkeit eines Arzneimittels nachweisen?" antworteten 120 Allgemeinmediziner und HNO-Ärzte.

\section{EbM und Phytotherapie - das passt}

Soweit so gut. Doch gelten dieselben Motive auch bei der Empfehlung von Phytotherapeutika oder stehen hier alleine Verträglichkeit, Patientenwunsch und Praxisbudget im Fokus der Überlegungen? Wohl kaum. Vielmehr erhielt ein in derselben Umfrage vorgestelltes Konzept zur Evidenzbasierung der Phytotherapie hohe Zustimmungswerte. 94\% der Teilnehmer sind der Meinung, dass Phytotherapie EbM sein kann, 53\% unterstützen diese Aussage sogar "auf jeden Fall".

Das Fazit der Befragung: Für die Verordnung von Arzneimitteln ist die Wirksamkeit nach EbM-Kriterien für Ärzte entscheidend. Darunter verstehen sie in der Mehrheit die Integration der gegenwärtig besten externen, wissenschaftlichen Evidenz mit der Erfahrung des behandelnden Arztes. Die Phytotherapie macht hier keine Ausnahme; auch sie kann nach Einschätzung der Ärzte evidenzbasierte Medizin sein - vorausgesetzt, Wirksamkeit, Erfahrung, Quali-

- Dr. Christoph Posch Quelle: Survey Konzept-Test, online gestützte Befragung von 60 Allgemeinmedizinern und 60 HNO-Ärzten, coliquio GmbH, Konstanz, September 2012
Internationaler Nitrolingual-Forschungspreis $\boldsymbol{\sim}$ Zur Förderung des wissenschaftlichen Dialogs um Glyceroltrinitrat lobt Pohl-Boskamp den mit 10000 Euro dotierten internationalen Nitrolingual ${ }^{\circledR}$-Forschungspreis 2013 aus. Der Goldstandard zur Behandlung eines Angina-pectorisAnfalls hat sich auch in der kardialen Rehabilitation etabliert: Glyceroltrinitrat steigert dosisabhängig die Angina-Schwelle, ermöglicht so ein effektives Training und überwindet Ängste von Reha-Patienten, sich an einem entsprechenden Sportprogramm zu beteiligen. Um den Preis bewerben können sich bis zum 1. Mai 2013 Nachwuchswissenschaftler der Fachbereiche Medizin und Naturwissenschaften mit unveröffentlichten Arbeiten über neue pharmakologische und klinische Erkenntnisse zu Glyceroltrinitrat (Einsenden an: Dr. med.Thomas Wittig, E-Mail: t.wittig@ pohl-boskamp.de, weitere Details unter www.pohl-boskamp.de).

Pohl Boskamp

Als Betroffener Fragen zu HIV? Heute gibt es zwar unendlich viele Informationen zur HIV-Erkrankung, doch Betroffene und Interessierte haben oft ganz spezifische Fragen. Helfen kann hier my-micromacro.net, das erste individuelle HIV-Portal, das mit einem intelligent programmierten elektronischen Filter („Wizzard“) arbeitet. Per Mausklick macht der User - der natürlich anonym bleibt - Angaben zu seiner Person, schon zeigt das Portal die relevanten Infos an.

Konkrete Fragen können auch direkt an drei HIV-Experten gestellt werden (Schwerpunkte "Infektiologie und suchtmedizinische Grundversorgung, insbesondere bei Frauen", "Psyche" und "medikamentöse Therapie") . Ein bundesweiter Adressfinder bietet darüber hinaus einen schnellen Überblick über HIV-Behandler, Schwerpunkt-Apotheken, Beratungs- sowie PEP-Stellen. Janssen Cilag 